\title{
MEG3/MIR-376B-3P/HMGA2 axis is involved in pituitary tumor invasiveness
}

\author{
*Dimin Zhu, MM,' Zheng Xiao, PhD,' Zongming Wang, PhD,, Bin Hu, PhD, ${ }^{1}$ Chengbin Duan, MM,1 \\ Ziyan Zhu, MB, ${ }^{2}$ Nailin Gao, MB, ${ }^{1}$ Yonghong Zhu, PhD, ${ }^{2}$ and Haijun Wang, $\mathrm{PhD}^{1}$ \\ 1Department of Neurosurgery and Pituitary Tumor Center, The First Affiliated Hospital of Sun Yat-sen University; and \\ ${ }^{2}$ Department of Histology and Embryology, Medical School of Sun Yat-sen University, Guangzhou, Guangdong, China
}

\begin{abstract}
OBJECTIVE To date, long noncoding RNAs (IncRNAs) have proven to function as key regulators in tumorigenesis. Among these IncRNAs, MEG3 displays low levels in various neoplasms and tumor cell lines. However, the regulatory mechanism of MEG3 and MIR-376B-3P, one of the microRNAs from downstream gene clusters of the DLK1-MEG3 locus, remains insufficiently defined.

METHODS The authors used quantitative real-time polymerase chain reaction analysis to analyze whether decreased MEG3 and MIR-376B-3P expression levels were associated with the invasiveness of clinical nonfunctioning pituitary adenomas (CNFPAs) in 30 patients. Furthermore, functional experiments unveiled the pathophysiological role of MEG3, MIR-376B-3P, and HMGA2 in pituitary-derived folliculostellate (PDFS) cell lines. Moreover, dual-luciferase reporter assay, Western blot analysis, and immunofluorescence were applied to reveal the correlations among MEG3, MIR-376B$3 P$, and $H M G A 2$.

RESULTS MEG3 and MIR-376B-3P were decreased in patients with CNFPA, and their transcriptional levels were highly associated with invasive CNFPAs. Moreover, excessive expression of MEG3 and MIR-376B-3P inhibited tumorigenesis and promoted apoptosis in PDFS cells. Importantly, the authors found that MEG3 acted as an enhancer of MIR-376B-3P expression. Furthermore, as a target gene of MIR-376B-3P, HMGA2 served as an oncogene in pituitary adenoma and could be negatively regulated by MEG3 via enriching MIR-376B-3P.
\end{abstract}

CONCLUSIONS This study offers a novel mechanism of an MEG3/MIR-376B-3P/HMGA2 regulatory network in CNFPAs, which may become a breakthrough for anticancer treatments.

https://thejns.org/doi/abs/10.3171/2019.10.JNS191959

KEYWORDS MEG3; IncRNA; MIR-376B-3P; pituitary adenoma; invasiveness; oncology; pituitary surgery

$\mathrm{P}$ ITUITARY adenoma (PA) is characterized by abnormal proliferation or hormone secretion of anterior glandular cells. The prevalence of PA ranges from 37.2 to 115.6 cases per 100,000 people. ${ }^{1,9,11,24,29} \mathrm{PA}$ is indicated by symptoms including headaches, visual decline, endocrinopathies, and even incidental lesions. ${ }^{19,21}$ Representing approximately one-third of all PA types, clinical nonfunctioning PAs (CNFPAs) are typically not related to obvious clinical and biochemical signs of hormonal hypersecretion. ${ }^{19}$ Additionally, neoplasm invasiveness in- fluences the outcome of surgery, medication, and radiotherapy. ${ }^{18}$

Growing evidence has demonstrated that the process of tumorigenesis occurs through a series of complex polygenic events. Despite the lack of coding capabilities, noncoding RNAs (ncRNAs) are vital elements in oncogenic activities., 3,27,31,39 Long ncRNAs (lncRNAs) contain more than 200 nucleotides and are customarily up to $100 \mathrm{~kb}$ in size, while microRNAs (miRNAs) are usually about 23 nucleotides long. Due to their intrinsic abilities, lncRNAs

ABBREVIATIONS CDNA = complementary DNA; CNFPA = clinical nonfunctioning PA; Dox = doxycycline; FBS = fetal bovine serum; FITC = fluorescein isothiocyanate; $\mathrm{HRP}=$ horseradish peroxidase; IncRNA = long ncRNA; miRNA = microRNA; NC = negative control; ncRNA = noncoding RNA; NP = normal pituitary; PA = pituitary adenoma; $P B S=$ phosphate-buffered saline; PDFS = pituitary-derived folliculostellate $; \mathrm{PI}=$ propidium iodide; $\mathrm{PVDF}=$ polyvinylidene fluoride; qRT-PCR = quantitative real-time polymerase chain reaction; TRITC = trimethylrhodamine isothiocyanate.

SUBMITTED July 21, 2019. ACCEPTED October 22, 2019.

INCLUDE WHEN CITING Published online January 3, 2020; DOI: 10.3171/2019.10.JNS191959.

* D.Z. and Z.X. contributed equally to this work. 
TABLE 1. Information of clinical patients $(n=30)$

\begin{tabular}{|c|c|c|c|c|}
\hline $\begin{array}{l}\text { Case } \\
\text { No. }\end{array}$ & Sex & $\begin{array}{l}\text { Age } \\
\text { (yrs) }\end{array}$ & $\begin{array}{l}\text { Max Diameter of Tumor } \\
\qquad(\mathrm{mm})\end{array}$ & $\begin{array}{l}\text { Knosp } \\
\text { Grade }\end{array}$ \\
\hline 1 & $M$ & 28 & 29 & 1 \\
\hline 2 & $F$ & 18 & 25 & 1 \\
\hline 3 & $F$ & 36 & 13 & 1 \\
\hline 4 & $F$ & 47 & 29 & 1 \\
\hline 5 & $M$ & 38 & 24 & 1 \\
\hline 6 & $M$ & 46 & 11 & 1 \\
\hline 7 & $F$ & 54 & 30 & 2 \\
\hline 8 & $M$ & 46 & 18 & 2 \\
\hline 9 & $F$ & 57 & 39 & 2 \\
\hline 10 & $F$ & 61 & 23 & 2 \\
\hline 11 & $F$ & 53 & 20 & 2 \\
\hline 12 & $M$ & 51 & 31 & 2 \\
\hline 13 & $\mathrm{~F}$ & 59 & 34 & 3 \\
\hline 14 & $M$ & 42 & 30 & 3 \\
\hline 15 & $M$ & 56 & 29 & 3 \\
\hline 16 & $M$ & 72 & 35 & 3 \\
\hline 17 & $F$ & 60 & 32 & 3 \\
\hline 18 & $F$ & 45 & 27 & 3 \\
\hline 19 & $M$ & 52 & 26 & 3 \\
\hline 20 & $F$ & 58 & 23 & 3 \\
\hline 21 & $F$ & 33 & 22 & 3 \\
\hline 22 & $F$ & 76 & 28 & 3 \\
\hline 23 & $F$ & 59 & 37 & 4 \\
\hline 24 & $M$ & 49 & 24 & 4 \\
\hline 25 & $M$ & 38 & 35 & 4 \\
\hline 26 & $F$ & 46 & 46 & 4 \\
\hline 27 & $F$ & 62 & 37 & 4 \\
\hline 28 & $M$ & 53 & 52 & 4 \\
\hline 29 & $F$ & 34 & 20 & 4 \\
\hline 30 & $F$ & 55 & 31 & 4 \\
\hline
\end{tabular}

regulate biological processes through interacting with DNA, RNA, and proteins. Additionally, miRNAs have the ability to match the $3^{\prime}$-UTR sequence of certain genes to suppress their expression. ${ }^{12}$

As an imprinted gene in the DLK1-MEG3 locus, $M E G 3$ has been identified as a tumor suppressor in $\mathrm{PA}^{34,35}$ and other types of tumors. ${ }^{8,13,16,33}$ Interestingly, selective suppression of the DLK1-MEG3 locus has been shown in human CNFPAs. ${ }^{17,36}$ The miRNA cluster in the DLK1MEG3 locus consists of more than 40 miRNAs. ${ }^{25}$ Among these miRNAs, the MIR-376 family exhibits a low transcriptional level similar to that of $M E G 3$ in neoplasms. ${ }^{2,4}$ However, the correlation between them has yet to be investigated.

In this study, we found that transcriptional levels of MEG3 and MIR-376B-3P had connections with tumor invasiveness in CNFPA patients. Then, we also confirmed that $M E G 3$ and $M I R-376 B-3 P$ could serve as tumor suppressor genes during proliferation of a pituitary-derived folliculostellate (PDFS) cell line. Moreover, MEG3 was found to be involved in $M I R-376 B-3 P$ expression regulation. We further validated that $M E G 3$ and $M I R-376 B-3 P$ could both downregulate $H M G A 2$. In summary, these data suggest that $M E G 3$ and $M I R-376 B-3 P$ synergistically regulate tumor invasiveness by targeting $H M G A 2$ and therefore serve as potential therapies for refractory CNFPAs.

\section{Methods \\ Clinical Samples}

In total, 30 patients with PA (Table 1) who underwent transsphenoidal surgery at our department were recruited into this study, and 12 normal pituitary (NP) tissues were provided by the Forensic Authentication Center of Sun Yat-sen University. Clinical invasiveness was defined by experienced neurosurgeons using the Knosp classification system $^{14}$ (noninvasive, $\mathrm{n}=12$; invasive, $\mathrm{n}=18$ ). All surgical specimens were clinically and pathologically confirmed as hormone-negative tumors. We also confirmed that the recruited patients who provided informed consent did not receive transsphenoidal surgery or other therapies prior to surgery. This study was authorized and supervised by the ethics committee.

\section{Cell Culture}

PDFS, a folliculostellate cell line, originates from a clinically nonfunctioning pituitary macroadenoma. ${ }^{7}$ PDFS exhibited no expression of hypophyseal hormone or Pit-1, which is a secretory pituitary cell marker. However, PDFS exhibited expression of vimentin and S100 protein, which is a folliculostellate-specific biomarker in the anterior pituitary gland. Due to the expression of activin A and follistatin, PDFS has an integral activin signaling pathway that regulates the autocrine/paracrine function of the anterior pituitary gland. Furthermore, PDFS can promote the proliferation of co-cultured primary pituitary tumor cells, and colony formation of this cell was found in soft agar? Therefore, PDFS is an ideal cell model for studying the biological behavior of CNFPAs. PDFS cells were maintained in DMEM (Invitrogen) supplemented with $10 \%$ fetal bovine serum (FBS; Invitrogen) and $1 \%$ antibiotics (Invitrogen). A human embryonic kidney cell line (HEK293T) was cultured in medium containing $10 \%$ FBS, nonessential amino acids, sodium pyruvate, and L-glutamine. Cells were cultured in $\mathrm{CO}_{2}$ incubator.

\section{Construction of Stable Cell Lines}

MEG3 plasmid construction and stable transfection were performed as previously described..$^{5}$ Briefly, for constructing an inducible $M E G 3$ transcription system, namely pTRE-MEG3, the cDNA sequence of $M E G 3$ was cloned into pTRE-Tight (ClonTech). In addition, pCMV-rtTA-puro was generated using the Tet-On system (ClonTech) as previously reported. ${ }^{5}$ Then, the above two plasmids were cotransfected into PDFS cells at a ratio of 3 to 1 using Lipofectamine 3000 reagent (Invitrogen). Successfully constructed cells were separated and propagated by puromycin selection. Transfection efficiency was then measured through quantitative real-time polymerase chain reaction (qRT-PCR) after adding doxycycline (Dox) to induce $M E G 3$ expression. 


\section{Cell Transfection}

PDFS cells $\left(1 \times 10^{6}\right.$ cells/well $)$ were grown in 6-well plates until attaining $60 \%-70 \%$ density. Then, we transfected $H s a-M I R-376 B-3 P$ mimics, Hsa-MIR-376B-3P inhibitor, si-HMGA2-1, si-HMGA2-2, and their corresponding blank reagent (RiboBio) into PDFS cells. Forty-eight hours later, qRT-PCR was performed to analyze the transfection efficiency.

\section{Quantitative Real-Time PCR}

TRIzol Reagent (Life Technologies) was used for RNA extraction. Synthesis of complementary DNA (cDNA) was conducted with the Prime Script RT Reagent Kit (Takara) and Mir-X miRNA First-Strand Synthesis Kit (Takara). The forward and reverse primers for MEG3 were 5'-CCTTCCATGCTGAGCT-3' and $5^{\prime}$-TGTTGGTGGGATCCAGGAAA-3', those for HMGA2 were 5'-CTACCTCAGCAGCAGTTGGA-3' and $5^{\prime}$-CCTGGGACTGTGAAGGGATT-3', the $5^{\prime}$ primer for $M I R-376 B-3 P$ was $5^{\prime}$-GCATCATAGAGGAAAATC CATG-3', and the corresponding $3^{\prime}$ primer was the mRQ $3^{\prime}$ primer supplied by the kit. The primers for U6 and GAPDH were synthesized by Sangon Biotech Co., Ltd. The qRT-PCR detection was used to determine the transcriptional level of certain genes.

\section{CCK-8 Assay}

Cell viability was monitored using a CCK-8 Kit (Sigma). The transfected PDFS cells were cultured in 96-well plates $\left(2 \times 10^{4}\right.$ cells/well). Ten microliters of CCK- 8 detection reagent was applied and incubated for 2-4 hours, followed by the detection of the 450-nm optical density value (microplate spectrophotometer).

\section{Flow Cytometry Assay}

The cell apoptotic rate was measured by an Annexin V-FITC/PI (fluorescein isothiocyanate/propidium iodide) Apoptosis Detection Kit (KeyGen Biotech). Specifically, 1 $\times 10^{5}$ treated cells were incubated in serum-free DMEM. Forty-eight hours later, $500 \mu \mathrm{l}$ of binding buffer was used to resuspend collected cells. After adding $5 \mu$ of Annexin V-FITC and $5 \mu \mathrm{l}$ of PI, cells were cultured and protected from light for 15 minutes. Stained cells were analyzed using a flow cytometer (CytoFLEX, Beckman Coulter).

\section{TUNEL Staining}

The treated cells were seeded on coverslips for at least 24 hours. After washing twice with phosphate-buffered saline (PBS), 4\% paraformaldehyde fixation was applied to treat cells for 15 minutes and $0.25 \%$ Triton-X 100 permeation for 20 minutes. TUNEL assay was performed in accordance with the kit's specification (KeyGen Biotech).

\section{Cell Colony Formation Assay}

PDFS cells $\left(1 \times 10^{2}\right.$ per well $)$ were cultured in a 6-well plate for at least 1 week after the transfection with corresponding reagent. The medium was replaced every 2 days. Cells were stained with crystal violet for 30 minutes after $4 \%$ formaldehyde fixation. The colonies (with a diameter $\geq 100 \mu \mathrm{m})$ were calculated to measure the proliferation ability of cells.

\section{Transwell Assay}

We used Matrigel chambers (BD Biosciences) to detect the cells' invasion ability. Briefly, transfected PDFS was seeded in FBS-free DMEM in the hydrated Matrigel chambers, while 10\% FBS was applied in the lower chambers. After 24 hours, invaded cells on the bottom surface were fixed and dyed with $0.1 \%$ crystal violet for 30 minutes. A microscope was used to count invaded cell numbers in three different fields.

\section{Wound Healing Assay}

A straight scratch was slightly scored on each treated cell monolayer in 6-well plates using a sterile Eppendorf tip. After washing away the detached cells with PBS buffer, FBS-free culture medium was added. Cells moving toward the scratch wound were monitored and imaged at $0,24,48$, and 72 hours using a microscope (Zeiss Axio Observer Z1).

\section{Luciferase Reporter Assay}

For HMGA2 3'-UTR analysis, the sequence containing regulatory sites of $M I R-376 B-3 P$ was synthesized and cloned into psiCHECK TM-2 vector (Promega). One hundred nanograms of constructed vector and $200 \mathrm{nmol} / \mathrm{L}$ MIR-376B-3P mimics or negative control (NC) were cotransfected into HEK-293T cells. A Dual-Luciferase Assay Kit (Promega) was used to detect cell lysate after 48 hours. We performed the experiment in triplicate.

\section{Immunohistochemistry and Immunofluorescence}

Paraffin-embedded slides of clinical adenomas and subcutaneous tumors were incubated with primary antibodies of HMGA2 (1:100, Cloud-Clone) and Ki-67 (1:50, Cloud-Clone) at $4^{\circ} \mathrm{C}$ overnight after the processes of dewaxing, dehydration, endogenous peroxidase suppression, and blocking. The slides were treated with biotinlabeled immunoglobulin $\mathrm{G}$ (CWBIO) for 10 minutes on the 2nd day. Streptavidin-horseradish peroxidase (HRP) and $\mathrm{DAB}$ dye liquor (CWBIO) were used sequentially to treat samples. Finally, sections were photographed under a microscope (Olympus BX63). Treated cells on coverslips were subjected to immunofluorescence staining after a similar fixation process. Then, the fixed cells were processed with $0.1 \%$ Triton X-100 and 5\% bovine serum albumin buffer for 10 and 30 minutes, respectively. The coverslips were treated with HMGA2 primary antibody (1:10, Cloud-Clone) before incubating with trimethylrhodamine isothiocyanate (TRITC)-conjugated anti-rabbit secondary antibody (KeyGen Biotech). DAPI was used for nuclear counterstaining, and images were captured using an Olympus camera (Olympus BX63).

\section{Western Blot Analysis}

Proteins were isolated using lysis buffer (Beyotime) with the addition of $1 \%$ phenylmethylsulfonyl fluoride (Amresco). Twelve percent sodium dodecyl sulfate- 
polyacrylamide gel electrophoresis was used to separate proteins $(50 \mu \mathrm{g})$ under constant voltage, followed by the transfer of samples onto polyvinylidene fluoride (PVDF) membranes (Bio-Rad). We blocked PVDF with 5\% defatted milk before incubating rabbit polyclonal antibodies to HMGA2 (1:1000, Cloud-Clone), BAX (1:2500, Proteintech), C-MYC (1:2000, Proteintech), BCL2 (1:2000, Proteintech), and GAPDH (1:5000, Cloud-Clone) at $4{ }^{\circ} \mathrm{C}$ overnight. We used Tris-buffered saline-Tween solution to wash the membranes 3 times and treated them with secondary antibody (1:3000, Proteintech) on the following day. PVDF membranes were photographed covered by Chemiluminescent HRP Substrate (Millipore Corporation) using the Amersham Imager 600 (GE).

\section{Tumor Xenograft In Vivo}

Forty-five 4-week-old nude mice and treated PDFS cells were assigned to 9 groups: 1) M-NC group (NC plasmid), 2) M-MEG3 group (MEG3 plasmid), 3) M-MIR-mimicsNC group (NC of mimics reagent), 4) M-MIR-mimics group (MIR-376B-3P mimics), 5) M-MIR-inhibitor-NC group (NC of inhibitor reagent), 6) M-MIR-inhibitor group (MIR-376B-3P inhibitor), 7) M-si-NC group (siNC), 8) M-si-1 group (si-HMGA2-1), and 9) M-si-2 group (si-HMGA2-2). Cell suspensions (200 $\mu 1,5 \times 10^{6}$ cells) of the abovementioned groups were injected subcutaneously into each mouse. Tumor parameters were measured with a digital caliper weekly. The volume (V) was calculated through assessing length (1) and width (w) as follows: $\mathrm{V}=$ $1 \mathrm{w}^{2} / 2$. The mice were killed and subcutaneous neoplasms were weighed on day 28. All animal research was verified by the Institutional Animal Ethics Committee and conducted under institutional guidelines.

\section{Statistical Analysis}

Statistical analysis was performed using SPSS software (version 20.0, IBM Corp.). Unless otherwise specified, differences between 2 and more than 2 groups were calculated using the Student t-test and 1-way ANOVA, respectively. A p value $<0.05$ was considered statistically significant $(* \mathrm{p}<0.05, * * \mathrm{p}<0.01, * * * \mathrm{p}<0.001, \mathrm{~ns}=$ no significance $)$.

\section{Results \\ MEG3 and MIR-376B-3P Expression Levels in CNFPA Patients}

In the current study, the expression levels of $M E G 3$ and $M I R-376 B-3 P$ in 30 CNFPAs and 12 NP tissues were determined. MEG3 expression was found to be decreased in CNFPAs compared to NP tissues (Fig. 1A). The transcription level of $M I R-376 B-3 P$, which belongs to the downstream gene cluster of $M E G 3$, was significantly different between CNFPAs and NP tissues (Fig. 1B). We also investigated the expression of other genes in the downstream gene cluster of MEG3 between CNFPAs and NP tissues and found that $M I R-376 B-3 P$ expression ranked the lowest among them (Fig. 1C). The average relative expression of $M E G 3$ was set as the margin, and 30 tumor tissues were segregated into high- and low-expression groups $(\mathrm{n}=8$ and 22, respectively). Correlations between MEG3 and the clinical parameters of patients were analyzed. As sug- gested in Supplemental Table 1, a low MEG3 level was closely connected with tumor invasiveness, but no statistical differences were found with indexes of age, sex, or tumor diameter. The comparison between the high $(\mathrm{n}=10)$ and low $(\mathrm{n}=20)$ expression groups of $M I R-376 B-3 P$ produced a similar result (Supplemental Table 2). Moreover, we defined tumor invasiveness using the Knosp classification system ${ }^{14}$ (Fig. 1D). Significantly lower transcriptional levels of $M E G 3$ and $M I R-376 B-3 P$ were determined in the noninvasive group compared to the invasive group (Fig. $1 \mathrm{E}$ and $\mathrm{F})$.

\section{MEG3 Overexpression, Cell Apoptosis, and Tumorigenesis In Vitro}

We supposed that disordered $M E G 3$ expression might have a bearing on tumor phenotype in CNFPAs on the basis of the aforementioned findings. We generated a stable clone of $M E G 3$ in PDFS cells as reported ${ }^{5}$ and named it PDFS-MEG3, and the negative control was named PDFS-NC. Overexpression efficiency was determined after treatment with Dox for 48 hours (Fig. 2A). A significant decline in cellular proliferation was detected in PDFS-MEG3 cells via CCK-8 assay (Fig. 2B). Next, we investigated cell apoptosis using flow cytometry analysis and TUNEL staining. A marked increase in apoptotic rate was shown in cells overexpressing MEG3 (Fig. 2C and D). Western blot analysis and cell colony formation assay showed similar results of a proliferative state with increased expression of apoptotic proteins and reduced colony formation in PDFS-MEG3 cells (Fig. 2E and F). Migration ability was also suppressed in PDFS-MEG3 (Fig. 2G). Moreover, a significant decline of cell invasive ability was found following MEG3 overexpression (Fig. $2 \mathrm{H})$. All these assays reveal that MEG3 exerts antineoplastic effects in CNFPAs.

\section{Inhibitory Role of MIR-376B-3P in the CNFPA Cell Line}

We used miRNA mimics to elucidate the role of MIR376B-3P in CNFPAs. After 24 hours of transfection with miRNA reagents, qRT-PCR was performed to ascertain the transfection efficiency (Fig. 3A). Accordingly, CCK8 assay demonstrated that $M I R-376 B-3 P$ also exhibited an inhibitory role in PDFS cell vitality (Fig. 3B). As expected, increased $M I R-376 B-3 P$ expression promoted cell apoptosis (Fig. 3C and D). Western blot analysis was used to determine BAX, BCL2, and C-MYC levels (Fig. 3E). Similarly, cell colony formation assay revealed that MIR$376 B-3 P$ could inhibit cell proliferation (Fig. 3F). Additionally, cell migration and invasiveness characteristics were inhibited in PDFS cells overexpressing $M I R-376 B$ $3 P$ (Fig. $3 \mathrm{G}$ and $\mathrm{H}$ ). As illustrated in Fig. 3, MIR-376B-3P functions as a suppressor similar to $M E G 3$ in PDFS cells.

\section{MEG3 and MIR-376B-3P Expression}

Given the tumor-suppressive roles of MEG3 and MIR$376 B-3 P$ in PDFS cells, we examined whether $M E G 3$ was involved in regulating $M I R-376 B-3 P$ expression. First, an increased $M I R-376 B-3 P$ transcriptional level was found in PDFS-MEG3 (Fig. 4A). As previously demonstrated, the $M E G 3$ promoter is hypermethylated in CNFPAs. ${ }^{36}$ There- 
A

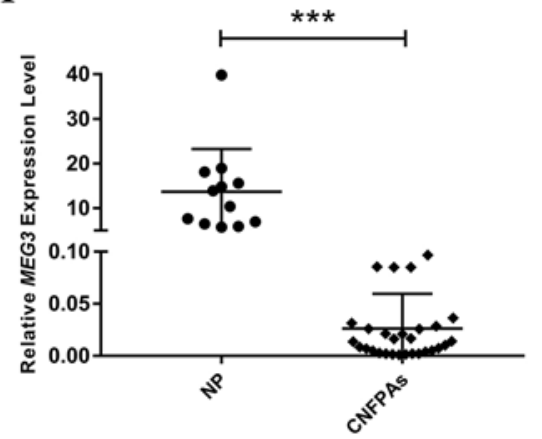

$\mathrm{B}$

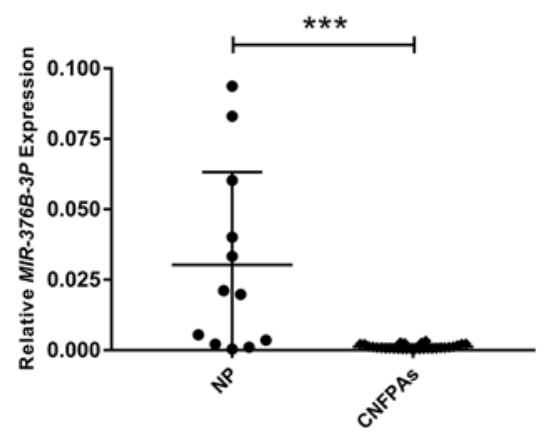

$\mathrm{C}$

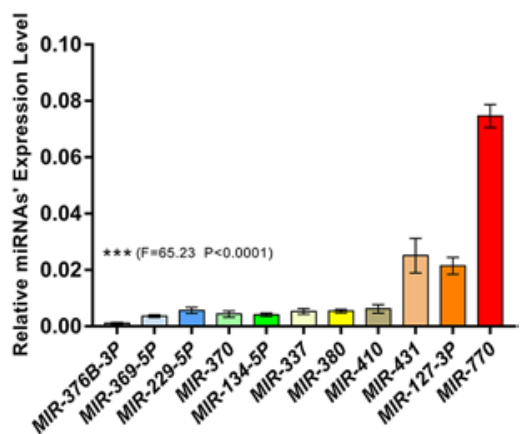

$\mathrm{E}$

$\mathrm{F}$

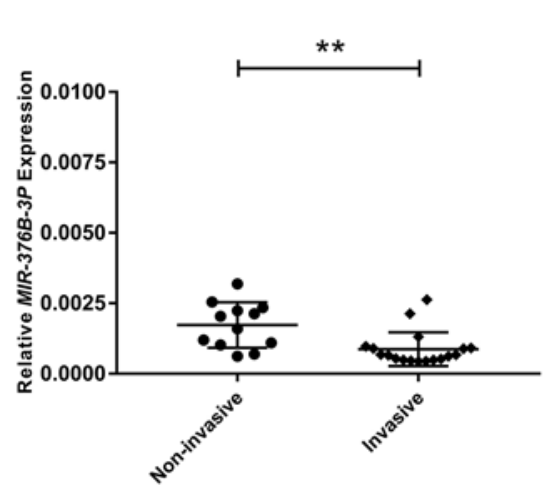

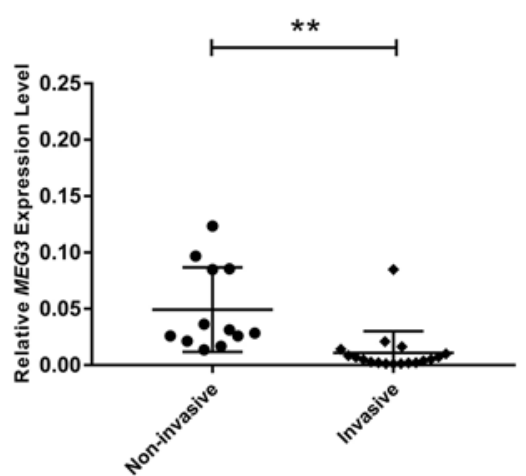

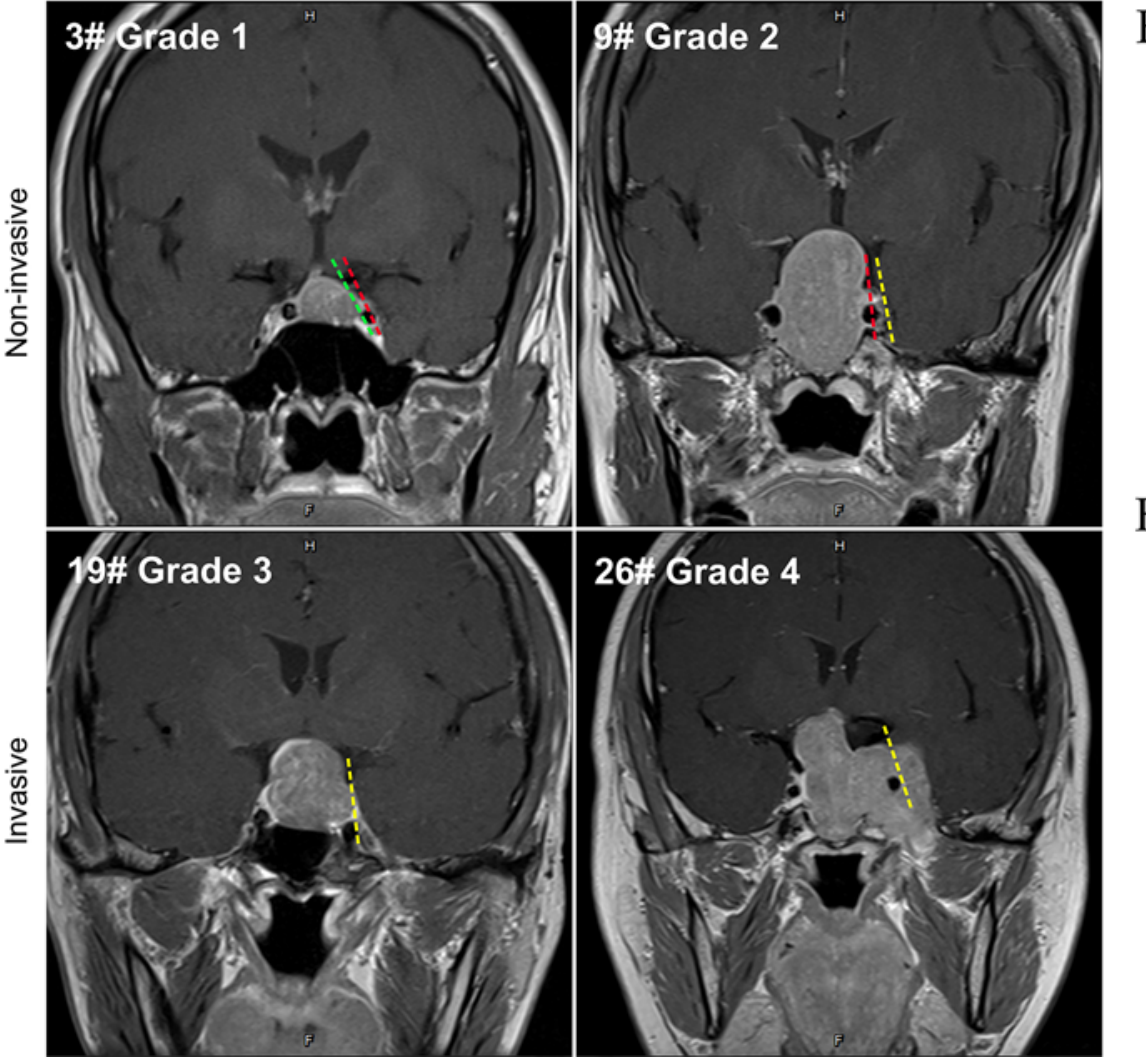

$\mathrm{D}$

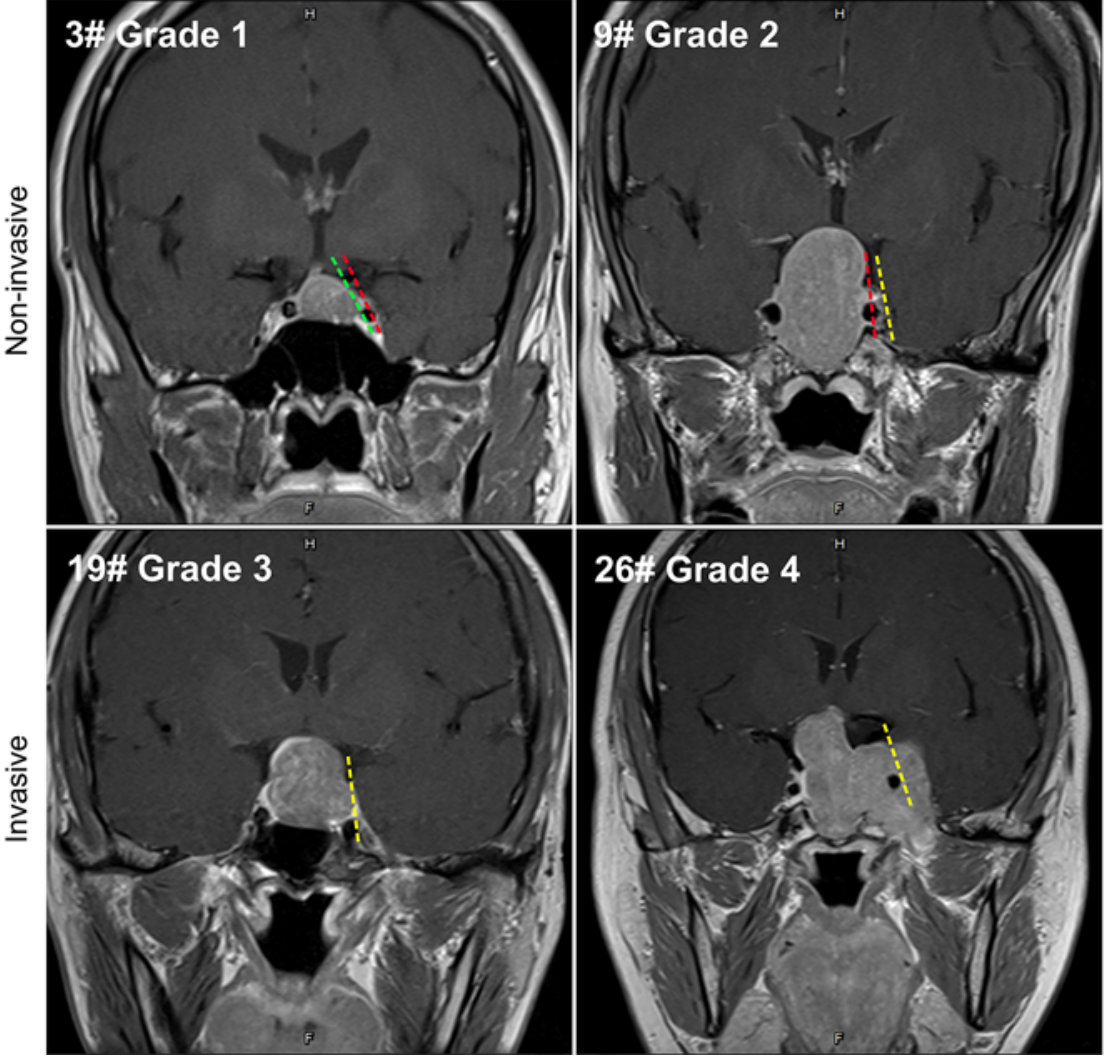

FIG. 1. MEG3 and MIR-376B-3P expression levels are decreased and correlate with invasiveness in patients with CNFPA.

A: The expression level of MEG3 was detected in 30 CNFPA patients and 12 NP tissues by qRT-PCR (Student t-test, $p<0.0001$ ).

B: Graph of the relative expression level of MIR-376B-3P between CNFPAs and NP tissues (Student t-test, $p<0.0001$ ). C: The expression levels of 11 miRNAs from downstream gene clusters of MEG3 (1-way ANOVA, F = 65.23, p < 0.0001). D: Coronal MRI in different patients (CNFPA cases 3, 9, 19, and 26). In grade 1, the tumor pushes into the medial wall of the cavernous sinus (green line) but does not go beyond a hypothetical line extending between the centers of the two segments of the internal carotid artery (red line). In grade 2, the tumor does not pass a line tangent to the lateral margins of the artery (yellow line). In grade 3, the tumor extends laterally to the internal carotid artery (yellow line) within the cavernous sinus. Grade 4 involves total encasement of the intracavernous internal carotid artery. E and F: Relative expression levels of MEG3 (Student t-test, $p=0.001$ ) and MIR-376B$3 P$ (Student t-test, $p=0.0025$ ) were analyzed in 12 noninvasive and 18 invasive CNFPAs. Data of all quantitative results are presented as the mean \pm SEM for three independent experiments. ${ }^{* *} p<0.01,{ }^{* *} p<0.001$. Figure is available in color online only.

fore, the effect of 5-Aza-CdR, a DNA-demethylating agent, on MEG3 and MIR-376B-3P was evaluated. Under DNA-demethylating agent treatment, their expression levels were significantly increased (Fig. 4B). Interestingly, both $M I R-376 B-3 P$ mimics and the inhibitor could not significantly change the expression level of MEG3 (Fig.
4C). These findings illuminated a positive role for $M E G 3$ in the transcriptional regulation of MIR-376B-3P.

\section{MEG3 and the Transcriptional Activity of HMGA2}

In vivo and in vitro experiments indicated that $H M G A 2$ played an oncogenic role in PA (Supplemental Figs. 1 and 
A

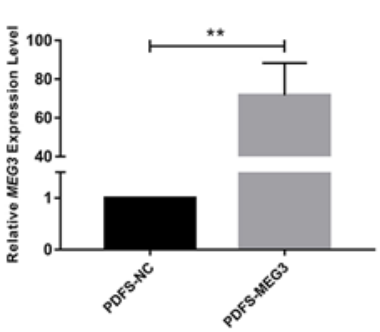

D

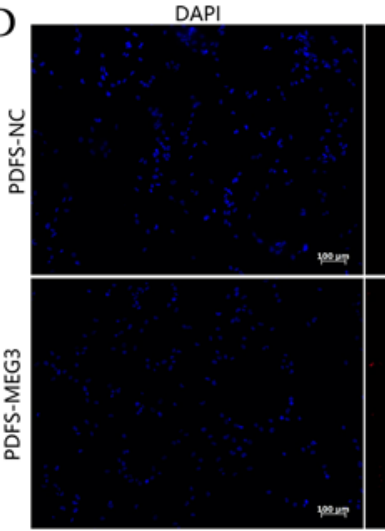

F
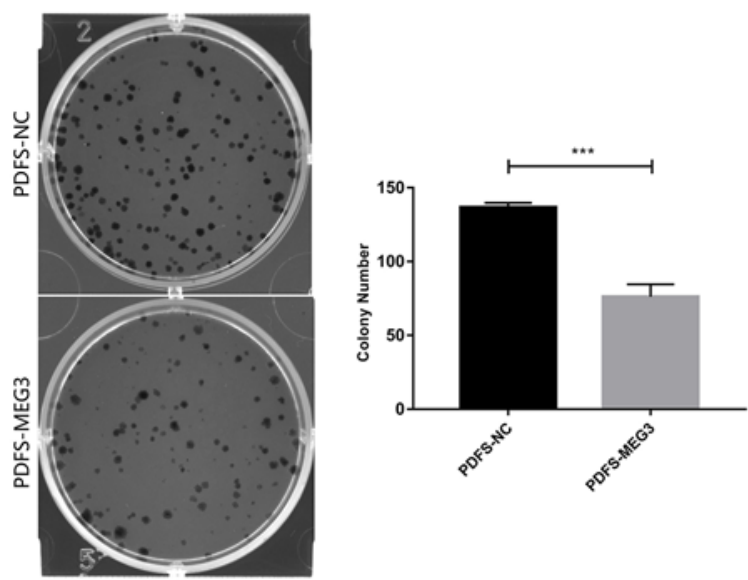

B

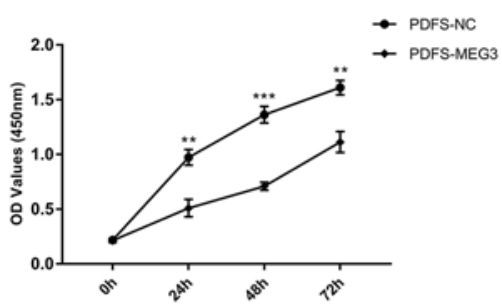

TUNEL

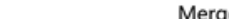

Merge
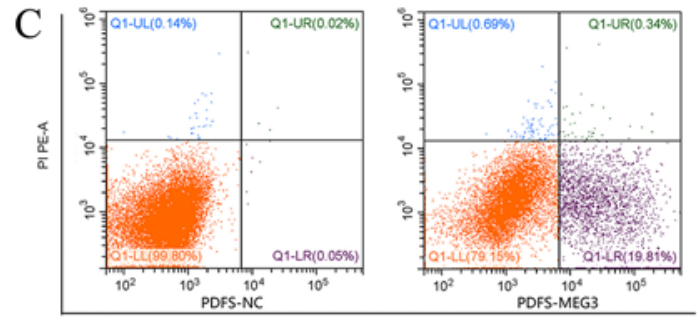

Annexin-V FITCA
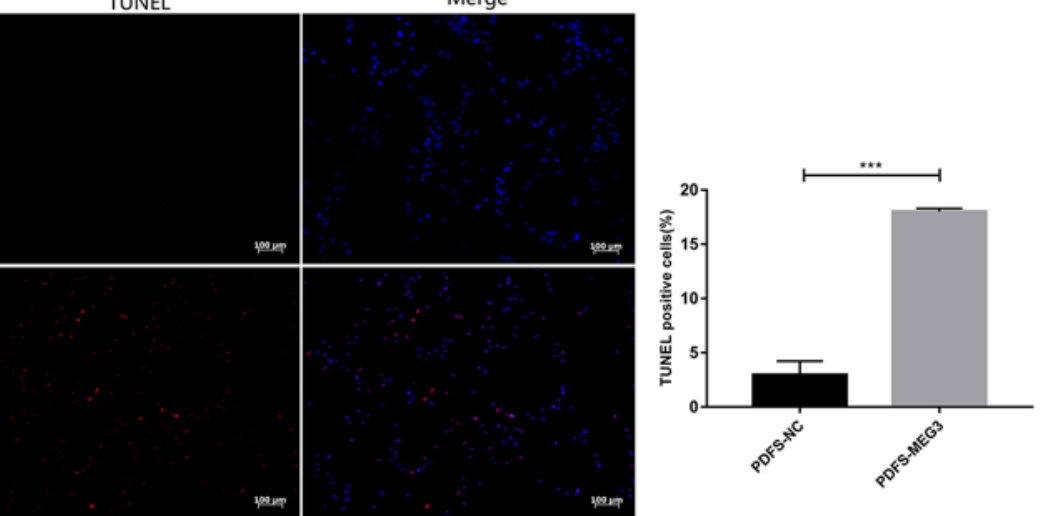

E

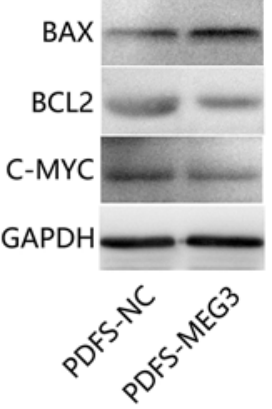

$\mathrm{H}$
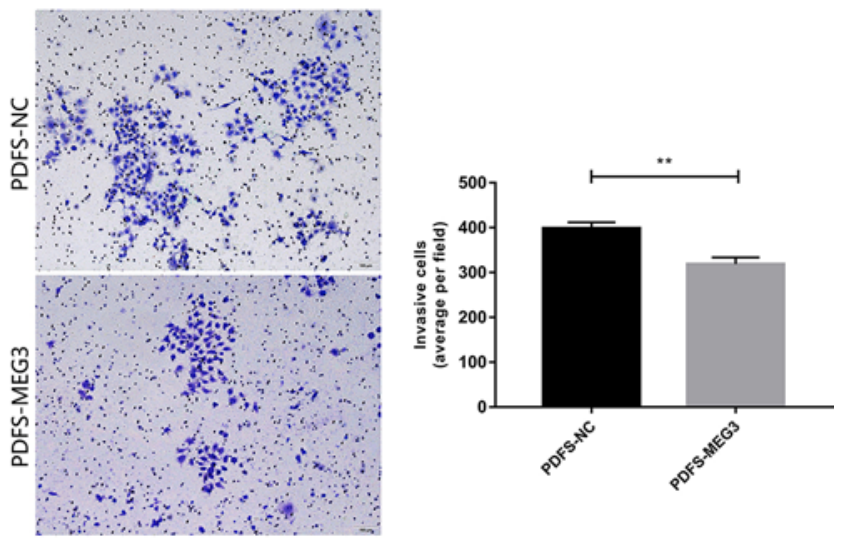

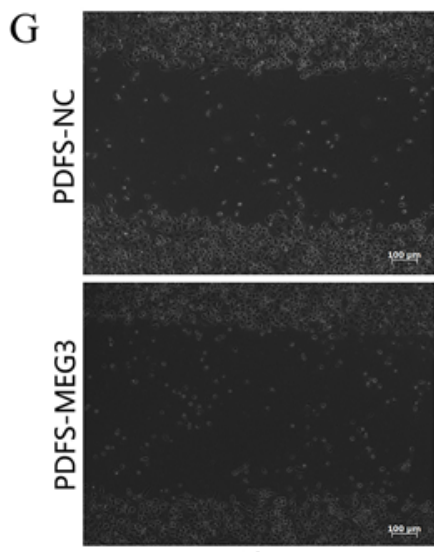

Oh

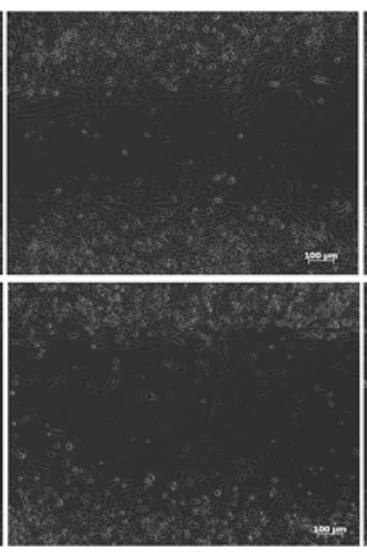

$24 \mathrm{~h}$

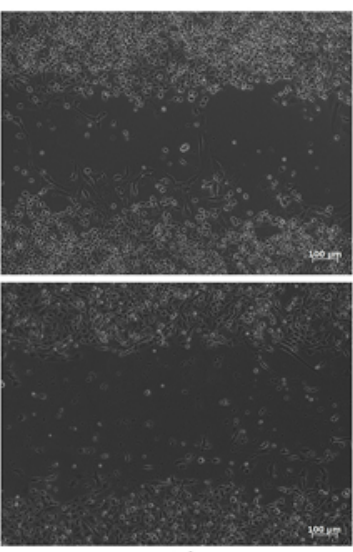

$48 \mathrm{~h}$

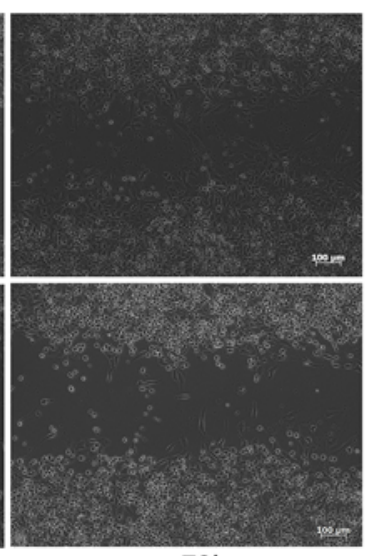

$72 \mathrm{~h}$

FIG. 2. MEG3 overexpression induces cell apoptosis and depresses tumorigenesis in vitro. A: The relative expression level of MEG3 in PDFS cells transfected with the corresponding plasmid. Data are presented as the fold change of PDFS-MEG3 to PDFS-NC cells (Student t-test, $p=0.0018$ ). B: Cell proliferation assay of PDFS-MEG3 and PDFS-NC cells after Dox treatment using CCK-8 (Student t-test, 24 hours, $p=0.0017 ; 48$ hours, $p=$ $0.0002 ; 72$ hours, $p=0.0017)$. FIG. 2. (continued) $\rightarrow$ 
FIG. 2. C: Flow cytometry was applied to examine the apoptosis of PDFS-MEG3 and PDFS-NC cells. FITC-A = strength of the FITC channel for detecting FITC-stained cells; PI PE-A = strength of phycoerythrin (PE) channel for detecting PI-stained cells; Q1-LL = lower-left quadrant, living cells; Q1-LR = lower-right quadrant, early apoptotic cells; Q1-UL = upper-left quadrant, necrotic cells; Q1-UR = upper-right quadrant, late apoptotic cells. D: TUNEL assay was used to detect the apoptotic effect of MEG3 in PDFS cells. DAPI was used to locate the nuclei; red cells indicated TUNEL-positive apoptotic cells (TUNEL stain, original magnification $\times 100$ ). Quantitative results of the TUNEL assay are shown on the graph (Student t-test, $p<0.0001)$. E: The protein levels of BAX, BCL2, and C-MYC in PDFS-MEG3 were detected by Western blot. F: Ectopic expression of MEG3 suppressed PDFS cell colony formation. The quantities of relative colony formation are shown on the graph (Student t-test, $p=0.0003$ ). G: Wound healing assays of PDFS-MEG3 and PDFS-NC cells were performed after Dox treatment (original magnification $\times 100)$. $\mathrm{H}$ : Transwell invasion assay indicated a significant reduction in cell invasiveness following MEG3 overexpression (crystal violet stain, original magnification $\times 100)$. The quantitative result is shown on the graph (Student t-test, $p=0.0021) .{ }^{* *} p<0.01,{ }^{* * *} p<0.001$. Figure is available in color online only.

2). Then, we observed that the upregulation of $H M G A 2$ was highly correlated with tumor invasiveness (Fig. 5A and B, Supplemental Table 3). Interestingly, using the online software TargetScan, we found that MIR-376B$3 P$ showed base complementarity with the 3 '-UTR of $H M G A 2$ (Fig. 5C). Then, a wild-type HMGA2 luciferase reporter (HMGA2-WT) and mutant HMGA2 luciferase reporter (HMGA2-MUT) were generated and transfected into HEK-293T cells to identify the regulatory relationship between MIR-376B-3P and HMGA2. Ectopic MIR-376B$3 P$ markedly decreased the fluorescence intensity of the wild-type vector but not that of the mutant one (Fig. 5D), which substantiates the possibility of a regulatory relationship between MIR-376B-3P and HMGA2.

Accordingly, we further investigated the $M E G 3 / M I R$ $376 B-3 P / H M G A 2$ axis. First, data showed that these two ncRNAs were negative regulators of $H M G A 2$ in both cells and clinical samples (Fig. 5E-G). Next, MIR-376B-3P mimics and inhibitor were introduced into PDFS-MEG3 cells, respectively. Interestingly, $M I R-376 B-3 P$ inhibition weakened the negative regulation of MEG3 on $H M G A 2$, whereas enhanced $M I R-376 B-3 P$ expression exerted an opposite effect (Fig. 5H and I). Moreover, Western blot analysis (Fig. 5J) and cyto-immunofluorescence (Fig. 5K) indicated that the presence of MEG3 and MIR-376B-3P could suppress $H M G A 2$ expression. These data indicated that $M E G 3$ can negatively regulate $H M G A 2$ by enhancing MIR-376B-3P expression.

\section{MEG3 and MIR-376B-3P, Tumorigenesis, and HMGA2 In Vivo}

To further explore the mechanisms of the $M E G 3 / M I R$ $376 B-3 P / H M G A 2$ axis in CNFPAs, the tumor formation experiment was performed to study their biofunctional role. Six groups of nude mice were established by hypodermic injection of cell suspension. Throughout tumor growth, mice injected with MEG3- and MIR-376B-3Ptransfected cells developed smaller tumor lumps than those from the negative control group (Fig. 6A). After 4 weeks, subcutaneous masses were resected carefully. As expected, we found that xenograft tumors from the MEG3 and $M I R-376 B-3 P$ groups were smaller and lighter than $\mathrm{NC}$ tumors (Fig. 6B and C). Moreover, MEG3 upregulated MIR-376B-3P expression, and downregulated HMGA2 at both transcriptional and translational levels (Fig. 6D and E). Furthermore, immunohistochemical analysis also showed that the Ki-67 index, which is a cellular marker for proliferation, was strongly reduced in the tumor tissues generated from MEG3- and MIR-376B-3P-transfected cells (Fig. 6F). Collectively, we concluded that upregulated
MEG3 and $M I R-376 B-3 P$ expression could inhibit tumor growth in vivo.

\section{Discussion}

Over the past decades, ncRNA has been an emerging hotspot in research on PAs. ${ }^{10,32,38} M E G 3$, the ortholog of mouse $G t l 2,{ }^{26}$ has been identified as a candidate antioncogene in human CNFPAs. ${ }^{37}$ We discovered that low $M E G 3$ expression had a connection with tumor invasiveness. Functional analyses confirmed the antitumor role of $M E G 3$ on PDFS cells. We further revealed that MEG3 contributed to negative downregulation of $H M G A 2$ via increasing the expression of $M I R-376 B-3 P$. Therefore, the current research reveals a new pathway, namely the $M E G 3 /$ $M I R-376 B-3 P / H M G A 2$ axis, which could shed light on the treatment of this difficult disease.

Mechanisms contributing to the regulatory crosstalk between gene clusters and their upstream host genes might be notable for uncovering CNFPA tumorigenesis. DNA methylation suppresses the expression of MIR-770 and MEG3, a host gene of the former, during neoplasm development..$^{13}$ In another study, the host gene MIR155HG served as a progression marker for patients with glioblastoma through interacting with $M I R-155 .{ }^{31}$ Furthermore, miRNAs can also target their upstream genes via binding with matching sequences. Instead of the regular 3'-UTR, MIR-932 regulated the transcription of its host gene $D N L G 2$, via base pairing to the coding sequence region. ${ }^{23}$ Interestingly, inconsistency between intronic miRNAs and their host genes was found to be quite common in hepatocellular carcinoma. ${ }^{28}$ The above studies show that the interactions between upand downstream genes are important in tumor formation. Therefore, we hypothesized that the regulatory network between $M E G 3$ and $M I R-376 B-3 P$ might also exhibit a vital part in CNFPA development. We observed a low transcriptional level of $M I R-376 B-3 P$, which was related to invasiveness, in pituitary tumor tissues. After performing functional experiments, we discovered that $M I R-376 B-3 P$ exerted an antitumor effect similar to that of MEG3. Moreover, our results supported the idea that $M E G 3$ overexpression induced MIR-376B-3P transcription.

Furthermore, it has been shown that miRNAs serve as suppressors by targeting mRNA in various tumors. In this study, online database analysis showed that $M I R-376 B-3 P$ shares common binding sites with $H M G A 2$ mRNA. Existing evidence has revealed that $H M G A 2$ is regarded as an oncogene in many cancers. With respect to PAs, regulatory networks including $H M G A 2$ may offer new insights for the exploration of treatments for precision medicine., ${ }^{6,20,22}$ 

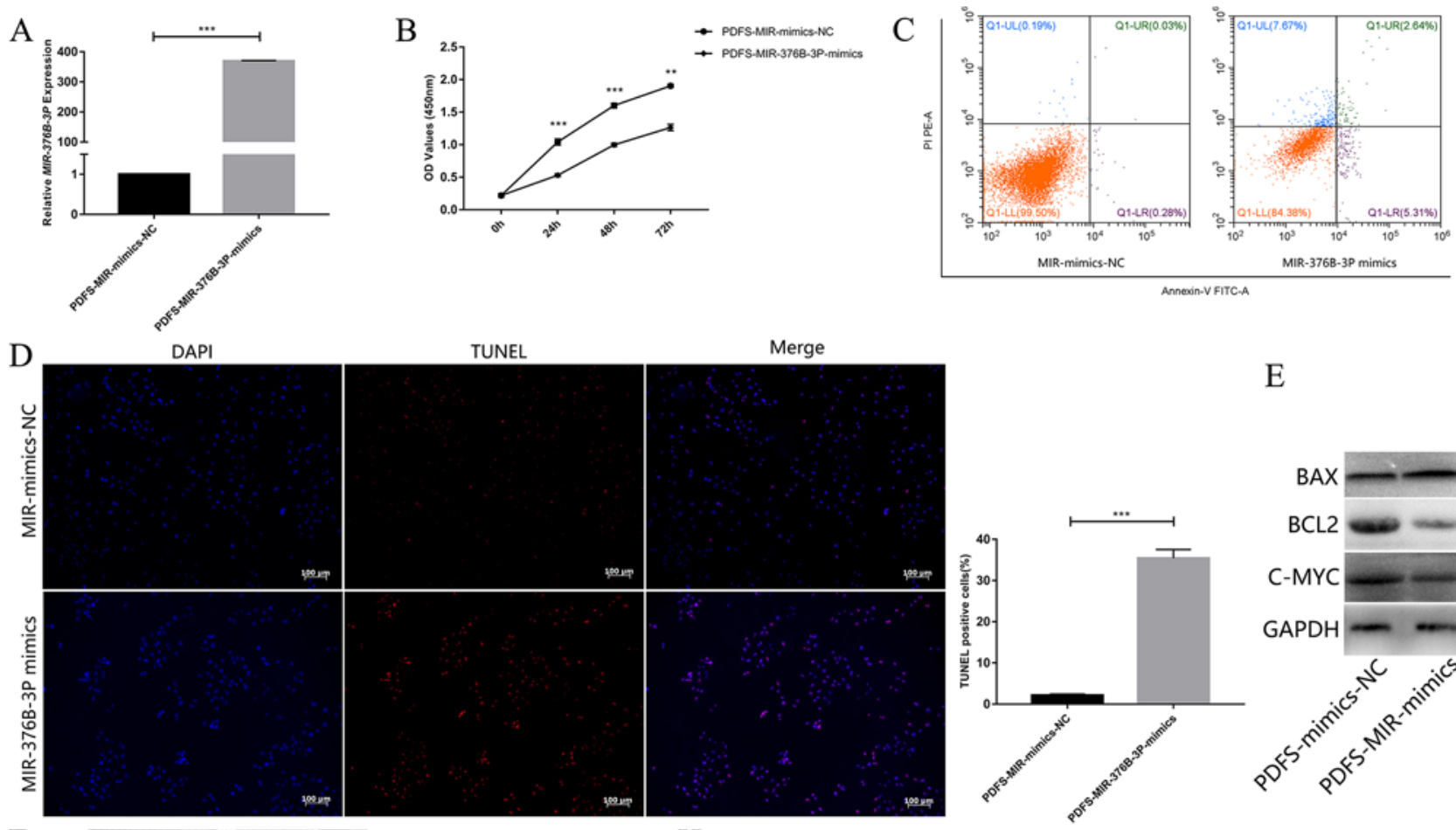

F

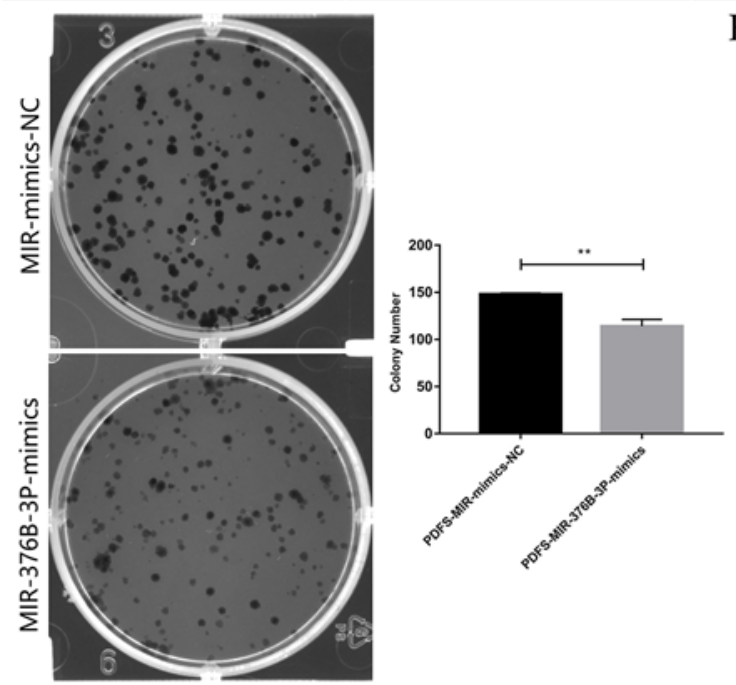

Merge

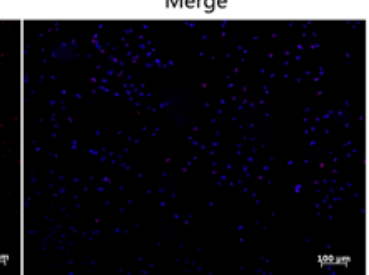

$100 \mathrm{~m}$

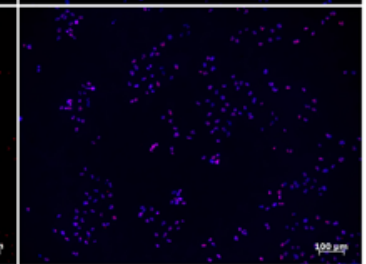

$\mathrm{H}$
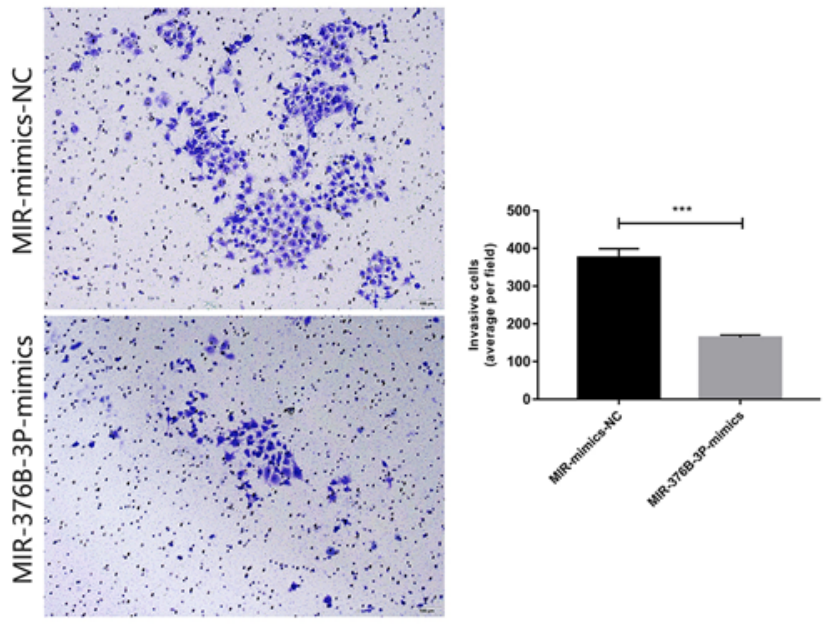

E

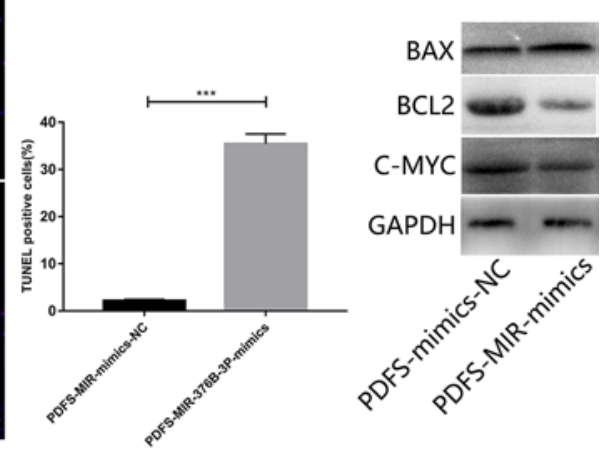

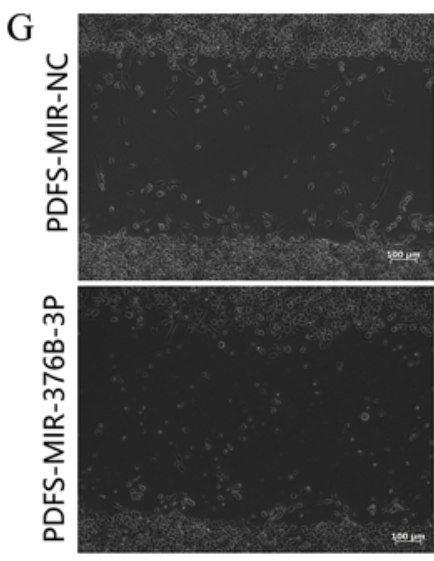

Oh
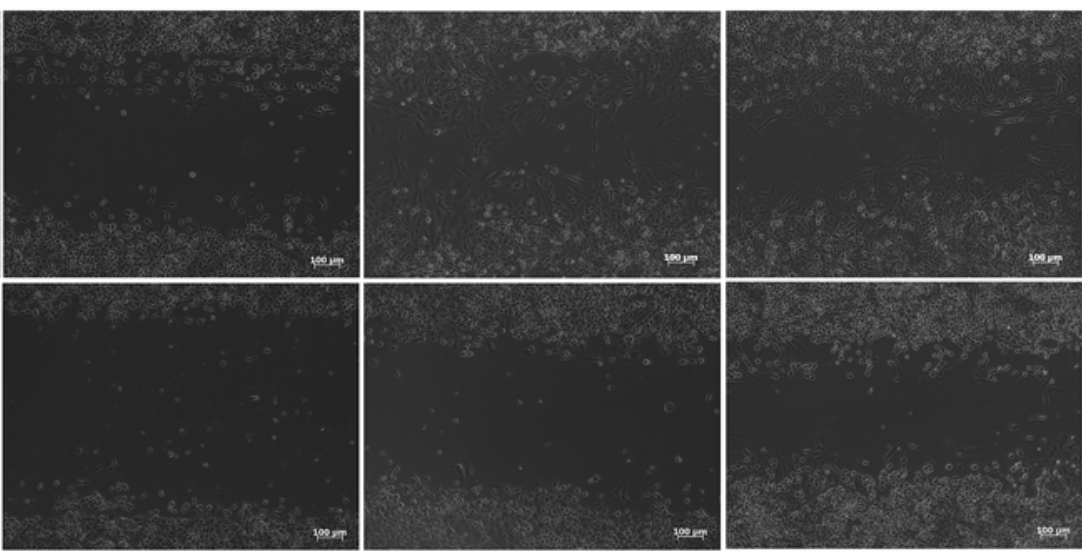

$24 \mathrm{~h}$

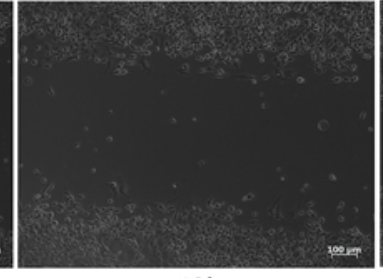

$48 \mathrm{~h}$

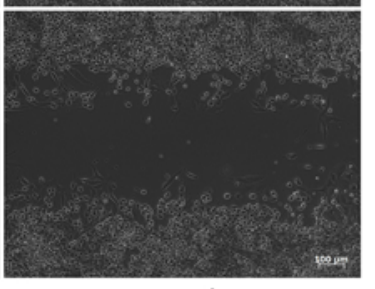

$72 \mathrm{~h}$

FIG. 3. MIR-376B-3P exhibits an inhibitory role similar to that of MEG3 in the CNFPA cell line. A: The relative expression level of MIR-376B-3P in PDFS cells transfected with miRNA mimics. Data are presented as the fold change of PDFS-MIR-376B-3P to PDFS-MIR-NC cells (Student t-test, $p<$ 0.0001). FIG. 3. (continued) $\rightarrow$ 
FIG. 3. B: Cell proliferation assay of PDFS cells was performed after transfection of MIR-376B-3P mimics using CCK-8 (Student t-test, 24 hours, $p=$ $0.0009 ; 48$ hours, $p=0.0005 ; 72$ hours, $p=0.0011)$. C: Flow cytometry was applied to examine the apoptotic rate of PDFS-MIR-376B-3P. D: TUNEL assay was used to detect apoptotic cells. DAPI was used to locate the nuclei; red cells indicate TUNEL-positive apoptotic cells (original magnification $\times 100$ ). Quantitative results of TUNEL assay are shown on the graph (Student t-test, $p<0.0001$ ). E: The protein levels of BAX, BCL2, and C-MYC in PDFS-MIR-376B-3P were detected by Western blot. F: Ectopic expression of MIR-376B-3P suppressed PDFS cell colony formation. The quantities of relative colony formation are shown on the graph (Student $t$-test, $p=0.0013$ ). G: Wound healing assays were performed after transfection (original magnification $\times 100$ ). $\mathrm{H}$ : Transwell invasion assay indicated that $M I R-376 B-3 P$ reduced cell invasiveness (crystal violet stain, original magnification $\times 100$ ). The quantitative result is shown on the graph (Student $t$-test, $p=0.0001$ ). ${ }^{* *} p<0.01,{ }^{* * *} p<0.001$. Figure is available in color online only.

Based on our findings, a hypothesis of whether the $M E G 3 /$ MIR-376B-3P/HMGA2 axis was involved in phenotypic regulation of CNFPAs had been proposed. We first validated that the presence of $M E G 3$ could enhance the efficiency of $M I R-376 B-3 P$ on the transcriptional regulation of $H M G A 2$. Next, enriching or silencing MIR-376B-3P expression could block or boost the expression of $H M G A 2$, which is regulated by the upstream gene $M E G 3$. Finally, we verified our analysis of this regulatory axis via in vivo experiments. In summary, our study affirmed that lncRNA
A

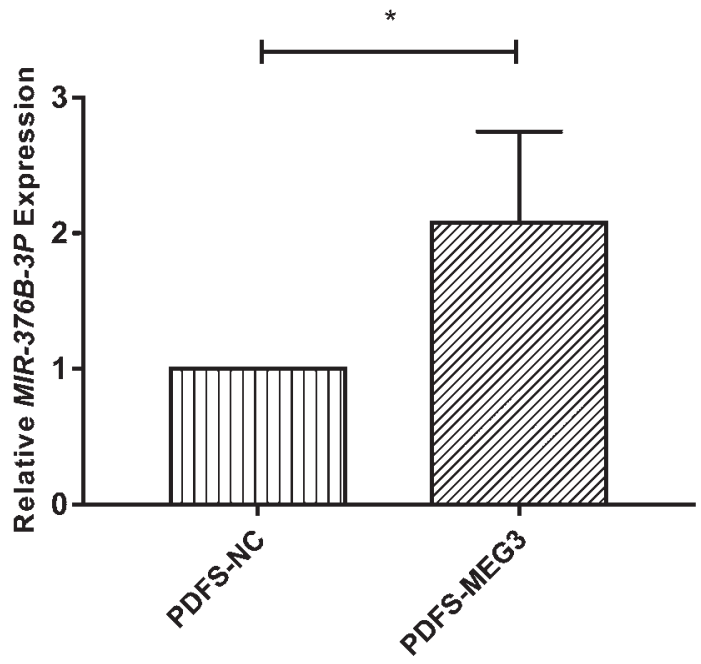

$\mathrm{C}$

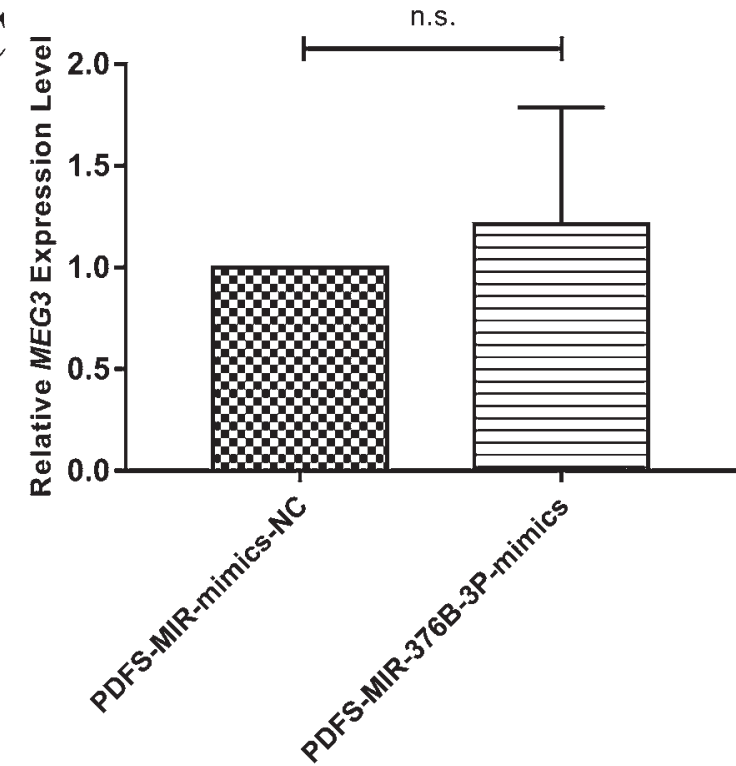

$\mathrm{B}$
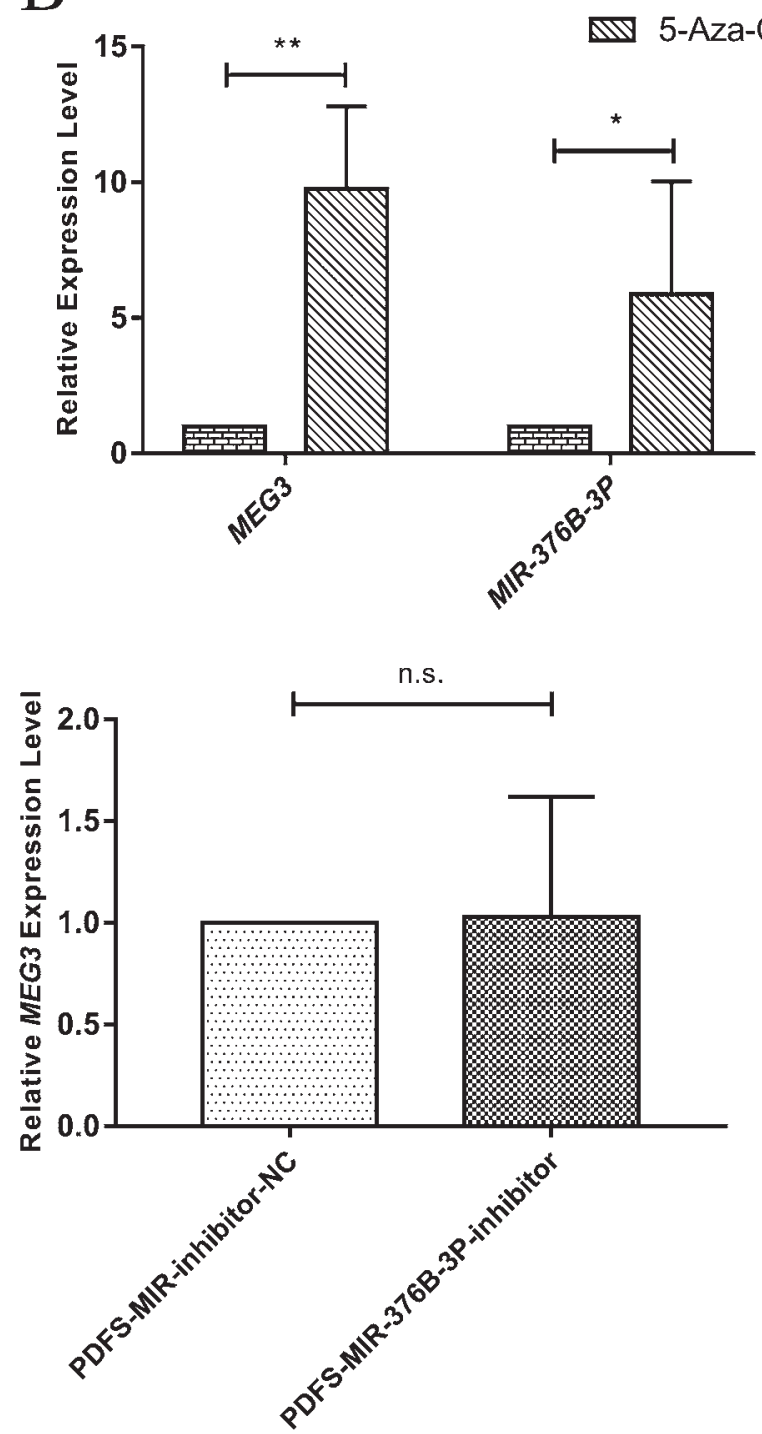

FIG. 4. MEG3 is involved in the expression of MIR-376B-3P. A: The relative expression level of MIR-376B-3P in PDFS cells overexpressing MEG3 (Student t-test, $p=0.032$ ). B: After 5-Aza-CdR treatment, the transcriptional levels of MEG3 (Student t-test, $p=$ 0.0012 ) and MIR-376B-3P (Student t-test, $p=0.0378$ ) were detected by qRT-PCR, and both showed similar tendencies following demethylation. C: The MEG3 expression level was analyzed by qRT-PCR after transfecting MIR-376B-3P mimics (Student t-test, $p$ $=0.3805$ ) or inhibitor (Student t-test, $p=0.8991$ ), both of which did not statistically change the transcriptional status of MEG3. ${ }^{*} p<$ $0.05,{ }^{* *} p<0.01$, n.s. $=$ no significance. 


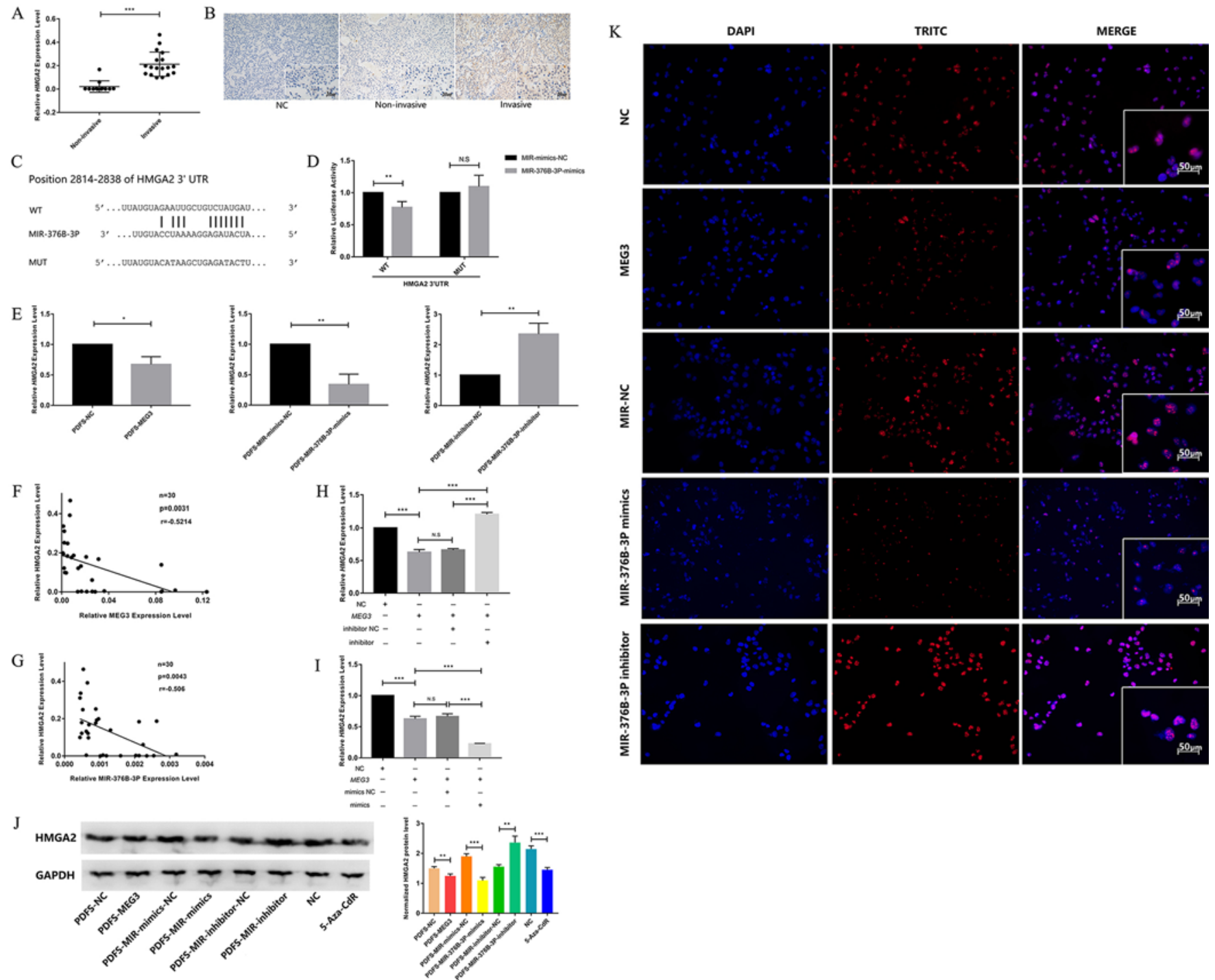

FIG. 5. MEG3 negatively regulates the transcriptional activity of HMGA2 by promoting MIR-376B-3P expression. A: The expression level of HMGA2 between noninvasive tumors and invasive neoplasms was detected via qRT-PCR (Student t-test, $p<$ 0.0001). B: Immunohistochemical analysis of HMGA2 regarding different biological characteristics of tumors (immunoperoxidase stain, original magnification $\times 200$ and $\times 400$ [inset]). C: The predicted binding sites of MIR-376B-3P on HMGA2 and the corresponding mutant sequence are shown. D: Luciferase reporter assay showed that MIR-376B-3P overexpression decreased the fluorescence activity in HEK-293T cells transfected with the HMGA2-WT (wild-type) vector (Student t-test, $p=0.0017$ ). Data are presented as the relative ratio of firefly luciferase activity to Renilla luciferase activity. MUT = mutant. E: The HMGA2 expression level was negatively correlated with MEG3 and MIR-376B-3P (Student t-test; left, center, and right: $p=0.0111, p=0.0025$, and $p=0.0028$, respectively). F: Pearson correlation analysis showed a significant negative correlation between MEG3 and HMGA2 (Pearson $r=-0.5214 ; p=0.0031, n=30)$. The line represents the linear regression of the data $\left(y=-1.994 x+0.1883 ; r^{2}=0.2719\right)$. G: Pearson correlation analysis showed a significant negative correlation between MIR-376B-3P and HMGA2 (Pearson $r=$ $-0.506 ; p=0.0043, n=30)$. The line represents the linear regression of the data $\left(y=-80.88 x+0.2343 ; r^{2}=0.256\right)$. H: Inhibition of MIR-376B-3P reversed the negative effect of MEG3 on HMGA2 (Student t-test; from left to right: $p=0.0001, p<0.0001, p<$ 0.0001). I: Enrichment of MIR-376B-3P enhanced the suppressive effect of MEG3 on HMGA2 (Student t-test; from left to right: $p=$ $0.0001, p<0.0001, p<0.0001)$. J: Immunoblot analysis of HMGA2 protein in PDFS cells under different treatments. Quantitative analysis of HMGA2 protein is shown on the graph (Student t-test; from left to right: $p=0.008, p=0.0005, p=0.0035, p=0.0009$ ). K: Immunofluorescence photomicrographs of HMGA2 immunostain-positive cells after different treatments. TRITC (red) indicates positive cells and DAPI (blue) shows nuclear counterstaining (original magnification $\times 100$ and $\times 200$ [inset]). ${ }^{*} p<0.05,{ }^{* *} p<0.01$, ${ }^{* * *} p<0.001, N . S=$ no significance. Figure is available in color online only.

MEG3 enriched downstream miRNA $M I R-376 B-3 P$ expression and thus negatively regulated $H M G A 2$.

Nevertheless, limitations of this study must also be acknowledged. In the latest classification of endocrine tu- mors, the pedigree of PAs has been emphasized. However, some commercially available antibodies are imperfect and exhibit unstable performance. Therefore, the patients enrolled in this study did not align with the classification 

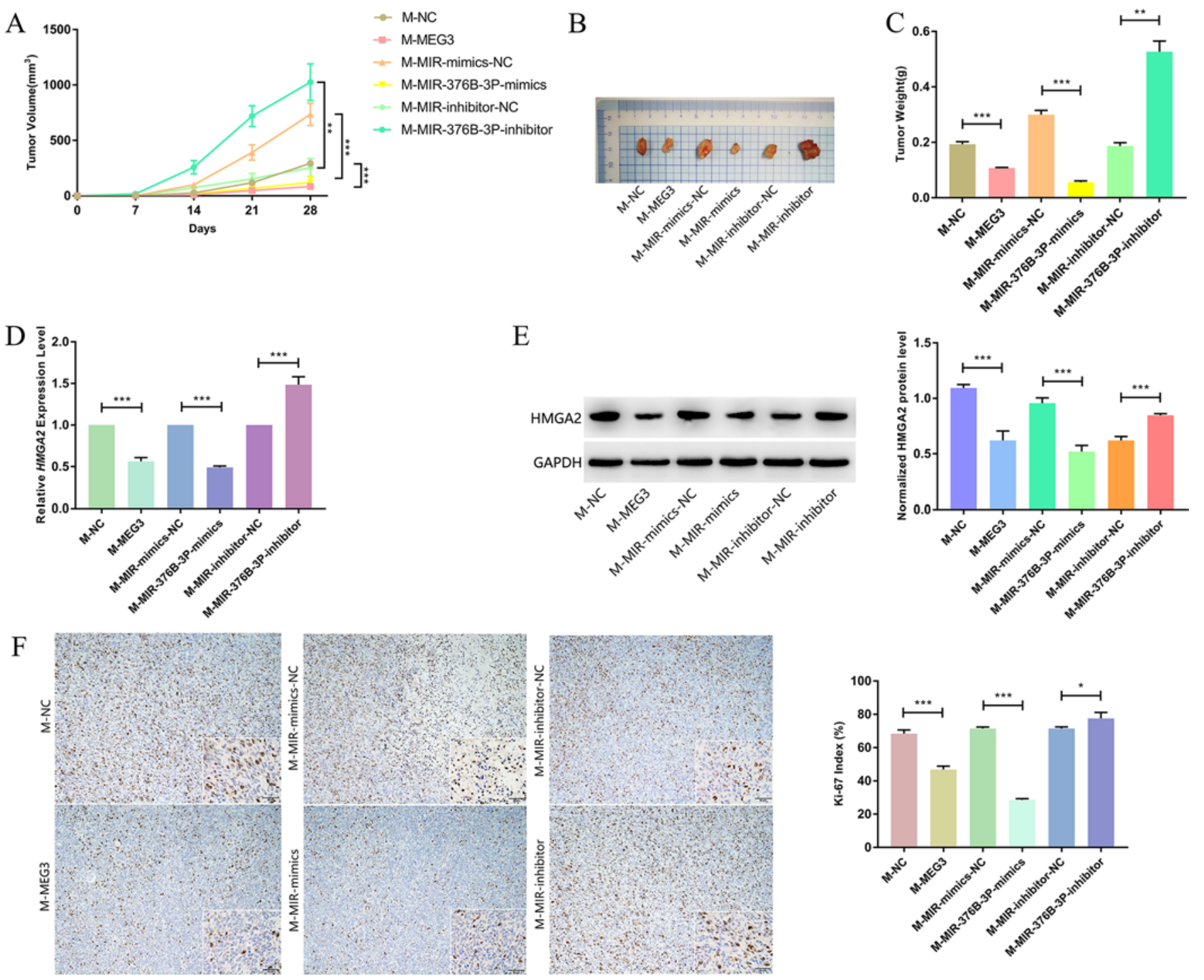

FIG. 6. MEG3 and MIR-376B-3P inhibit tumorigenesis by targeting HMGA2 in vivo. A: A tumor growth curve of experimental nude mouse models was generated following PDFS cell injection. Tumor volumes were calculated weekly (Student t-test; from top to bottom: $p=0.002, p=0.0007, p=0.0002$ ). B: Mouse tumor sizes at the end of 28 days. C: Tumor weights are represented as means \pm SEM (Student t-test; from left to right: $p=0.0007, p=0.0001, p=0.0011)$. $\mathbf{D}$ and $E$ : The transcriptional (Student t-test; from left to right: $p<0.0001, p<0.0001, p=0.0009$ ) and translational (Student t-test; from left to right: $p=0.0008, p=0.0005, p=$ 0.0006 ) levels of HMGA2 were measured after dissociating the obtained tumor samples. F: Immunohistochemistry assay of Ki-67 was conducted to detect the proliferative activity of different subcutaneous tumors (Student t-test; from left to right: $p=0.0003, p$ $<0.0001, p=0.0463$; immunoperoxidase stain, original magnification $\times 200$ and $x 400$ [inset]). ${ }^{*} p<0.05,{ }^{* *} p<0.01,{ }^{* * *} p<0.001$. Figure is available in color online only.

criteria. Although we discovered that $M E G 3$ enriched MIR-376B-3P expression, the specific underlying mechanisms are yet to be identified. It has been confirmed that lncRNA could be engaged in processing primary miRNA transcripts by sponging MIR-361 to relieve the inhibitory effect on pri-MIR-484 and promote Drosha-mediated processing into pre-MIR-484. ${ }^{30}$ Another study reported a similar effect of lncRNA on pri-miRNA processing. ${ }^{15}$ Hence, a complex network involving the processing of primary transcripts between MEG3 and MIR-376B-3P is conceptually plausible. Transcriptional factors regulated by $M E G 3$ might also interact with the enhancer region of MIR-376B-
$3 P$. The specific regulatory network between $M E G 3$ and $M I R-376 B-3 P$ needs to be comprehensively analyzed in future studies.

\section{Conclusions}

We present the first report on a novel mechanism of lncRNA $M E G 3$ as a negative regulator in $H M G A 2$ by upregulating the expression of $M I R-376 B-3 P$ to regulate invasiveness in CNFPAs (Fig. 7). Targeting the interaction of $M E G 3 / M I R-376 B-3 P / H M G A 2$ will aid in designing new strategies for therapeutic intervention in PAs. 


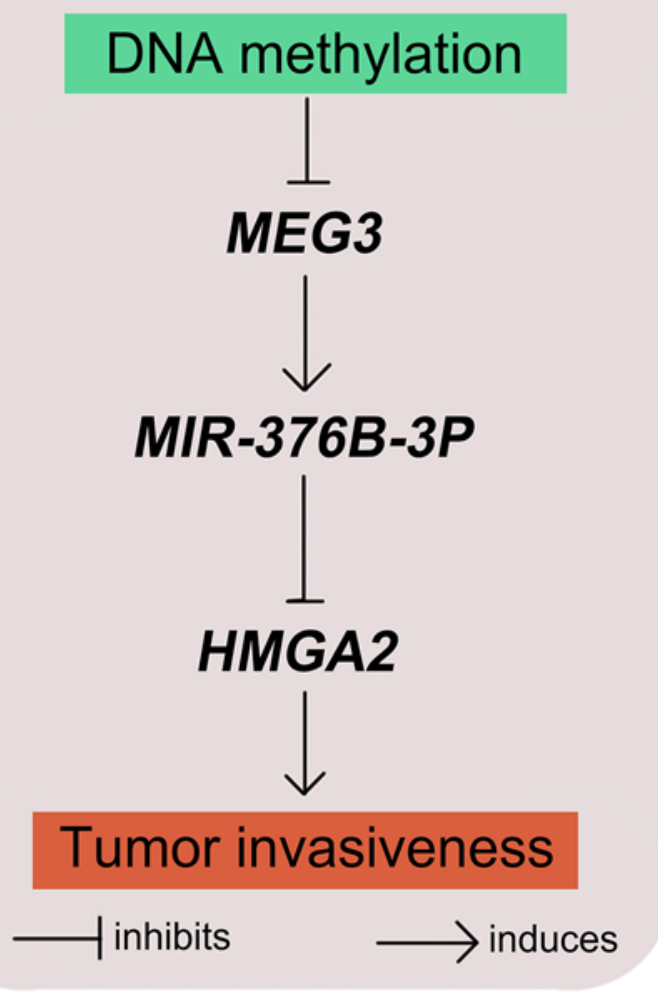

FIG. 7. Model for the relationship between the MEG3/MIR-376B-3P/ $H M G A 2$ axis and tumor invasiveness. DNA methylation negatively regulates MEG3, which induces the MIR-376B-3P mRNA level. MIR$376 B-3 P$ inhibits the HMGA2 expression level, thereby affecting pituitary tumor invasiveness. Figure is available in color online only.

\section{Acknowledgments}

Our research was supported by the Guangzhou Science and Technology Project (grant no. 201704020085), Scientific Research Project of Guangdong Traditional Chinese Medicine Bureau (grant no. 20173003), and Sun Yat-sen University Clinical Research 5010 Program (grant no. 2016008).

\section{References}

1. Agustsson TT, Baldvinsdottir T, Jonasson JG, Olafsdottir E, Steinthorsdottir V, Sigurdsson G, et al: The epidemiology of pituitary adenomas in Iceland, 1955-2012: a nationwide population-based study. Eur J Endocrinol 173:655-664, 2015

2. Butz H, Likó I, Czirják S, Igaz P, Korbonits M, Rácz K, et al: MicroRNA profile indicates downregulation of the TGF $\beta$ pathway in sporadic non-functioning pituitary adenomas. Pituitary 14:112-124, 2011

3. Chen ZH, Wang WT, Huang W, Fang K, Sun YM, Liu SR, et al: The lncRNA HOTAIRM1 regulates the degradation of PML-RARA oncoprotein and myeloid cell differentiation by enhancing the autophagy pathway. Cell Death Differ 24:212-224, 2017

4. Cheunsuchon P, Zhou Y, Zhang X, Lee H, Chen W, Nakayama Y, et al: Silencing of the imprinted DLK1-MEG3 locus in human clinically nonfunctioning pituitary adenomas. Am J Pathol 179:2120-2130, 2011

5. Chunharojrith P, Nakayama Y, Jiang X, Kery RE, Ma J, De La Hoz Ulloa CS, et al: Tumor suppression by MEG3 ln-
cRNA in a human pituitary tumor derived cell line. Mol Cell Endocrinol 416:27-35, 2015

6. D'Angelo D, Palmieri D, Mussnich P, Roche M, Wierinckx A, Raverot G, et al: Altered microRNA expression profile in human pituitary GH adenomas: down-regulation of miRNA targeting HMGA1, HMGA2, and E2F1. J Clin Endocrinol Metab 97:E1128-E1138, 2012

7. Danila DC, Zhang X, Zhou Y, Dickersin GR, Fletcher JA, Hedley-Whyte ET, et al: A human pituitary tumor-derived folliculostellate cell line. J Clin Endocrinol Metab 85:11801187,2000

8. Dong Z, Zhang A, Liu S, Lu F, Guo Y, Zhang G, et al: Aberrant methylation-mediated silencing of lncRNA MEG3 functions as a ceRNA in esophageal cancer. Mol Cancer Res 15:800-810, 2017

9. Fernandez A, Karavitaki N, Wass JA: Prevalence of pituitary adenomas: a community-based, cross-sectional study in Banbury (Oxfordshire, UK). Clin Endocrinol (Oxf) 72:377-382, 2010

10. Fu D, Zhang Y, Cui H: Long noncoding RNA CCAT2 is activated by $\mathrm{E} 2 \mathrm{~F} 1$ and exerts oncogenic properties by interacting with PTTG1 in pituitary adenomas. Am J Cancer Res 8:245-255, 2018

11. Gruppetta M, Mercieca C, Vassallo J: Prevalence and incidence of pituitary adenomas: a population based study in Malta. Pituitary 16:545-553, 2013

12. Guil S, Esteller M: RNA-RNA interactions in gene regulation: the coding and noncoding players. Trends Biochem Sci 40:248-256, 2015

13. Guo W, Dong Z, Liu S, Qiao Y, Kuang G, Guo Y, et al: Promoter hypermethylation-mediated downregulation of miR-770 and its host gene MEG3, a long non-coding RNA, in the development of gastric cardia adenocarcinoma. Mol Carcinog 56:1924-1934, 2017

14. Knosp E, Steiner E, Kitz K, Matula C: Pituitary adenomas with invasion of the cavernous sinus space: a magnetic resonance imaging classification compared with surgical findings. Neurosurgery 33:610-618, 1993

15. Liz J, Portela A, Soler M, Gómez A, Ling H, Michlewski G, et al: Regulation of pri-miRNA processing by a long noncoding RNA transcribed from an ultraconserved region. Mol Cell 55:138-147, 2014

16. Lyu Y, Lou J, Yang Y, Feng J, Hao Y, Huang S, et al: Dysfunction of the WT1-MEG3 signaling promotes AML leukemogenesis via p53-dependent and -independent pathways. Leukemia 31:2543-2551, 2017

17. Mezzomo LC, Gonzales PH, Pesce FG, Kretzmann Filho N, Ferreira NP, Oliveira MC, et al: Expression of cell growth negative regulators MEG3 and GADD45 $\gamma$ is lost in most sporadic human pituitary adenomas. Pituitary 15:420-427, 2012

18. Micko A, Oberndorfer J, Weninger WJ, Vila G, Höftberger R, Wolfsberger S, et al: Challenging Knosp high-grade pituitary adenomas. J Neurosurg [epub ahead of print May 31, 2019. DOI: 10.3171/2019.3.JNS19367]

19. Molitch ME: Diagnosis and treatment of pituitary adenomas: a review. JAMA 317:516-524, 2017

20. Palmieri D, Valentino T, De Martino I, Esposito F, Cappabianca P, Wierinckx A, et al: PIT1 upregulation by HMGA proteins has a role in pituitary tumorigenesis. Endocr Relat Cancer 19:123-135, 2012

21. Paschou SA, Vryonidou A, Goulis DG: Pituitary incidentalomas: a guide to assessment, treatment and follow-up. Maturitas 92:143-149, 2016

22. Pierantoni GM, Finelli P, Valtorta E, Giardino D, Rodeschini $\mathrm{O}$, Esposito F, et al: High-mobility group A2 gene expression is frequently induced in non-functioning pituitary adenomas (NFPAs), even in the absence of chromosome 12 polysomy. Endocr Relat Cancer 12:867-874, 2005 
23. Qian J, Tu R, Yuan L, Xie W: Intronic miR-932 targets the coding region of its host gene, Drosophila neuroligin2. Exp Cell Res 344:183-193, 2016

24. Raappana A, Koivukangas J, Ebeling T, Pirilä T: Incidence of pituitary adenomas in Northern Finland in 1992-2007. J Clin Endocrinol Metab 95:4268-4275, 2010

25. Royo H, Cavaillé J: Non-coding RNAs in imprinted gene clusters. Biol Cell 100:149-166, 2008

26. Schuster-Gossler K, Bilinski P, Sado T, Ferguson-Smith A, Gossler A: The mouse Gt12 gene is differentially expressed during embryonic development, encodes multiple alternatively spliced transcripts, and may act as an RNA. Dev Dyn 212:214-228, 1998

27. Stevic I, Müller V, Weber K, Fasching PA, Karn T, Marmé F, et al: Specific microRNA signatures in exosomes of triplenegative and HER2-positive breast cancer patients undergoing neoadjuvant therapy within the GeparSixto trial. BMC Med 16:179, 2018

28. Sun Y, Ji F, Kumar MR, Zheng X, Xiao Y, Liu N, et al: Transcriptome integration analysis in hepatocellular carcinoma reveals discordant intronic miRNA-host gene pairs in expression. Int J Biol Sci 13:1438-1449, 2017

29. Tjörnstrand A, Gunnarsson K, Evert M, Holmberg E, Ragnarsson $\mathrm{O}$, Rosén $\mathrm{T}$, et al: The incidence rate of pituitary adenomas in western Sweden for the period 2001-2011. Eur J Endocrinol 171:519-526, 2014

30. Wang K, Sun T, Li N, Wang Y, Wang JX, Zhou LY, et al: MDRL lncRNA regulates the processing of miR-484 primary transcript by targeting miR-361. PLoS Genet 10:e1004467, 2014

31. Wu X, Wang Y, Yu T, Nie E, Hu Q, Wu W, et al: Blocking MIR155HG/miR-155 axis inhibits mesenchymal transition in glioma. Neuro Oncol 19:1195-1205, 2017

32. Wu ZR, Yan L, Liu YT, Cao L, Guo YH, Zhang Y, et al: Inhibition of mTORC1 by lncRNA H19 via disrupting 4EBP1/Raptor interaction in pituitary tumours. Nat Commun 9:4624, 2018

33. Zhang X, Gejman R, Mahta A, Zhong Y, Rice KA, Zhou Y, et al: Maternally expressed gene 3, an imprinted noncoding RNA gene, is associated with meningioma pathogenesis and progression. Cancer Res 70:2350-2358, 2010

34. Zhang X, Rice K, Wang Y, Chen W, Zhong Y, Nakayama Y, et al: Maternally expressed gene 3 (MEG3) noncoding ribonucleic acid: isoform structure, expression, and functions. Endocrinology 151:939-947, 2010

35. Zhang X, Zhou Y, Mehta KR, Danila DC, Scolavino S, Johnson SR, et al: A pituitary-derived MEG3 isoform functions as a growth suppressor in tumor cells. J Clin Endocrinol Metab 88:5119-5126, 2003

36. Zhao J, Dahle D, Zhou Y, Zhang X, Klibanski A: Hypermethylation of the promoter region is associated with the loss of MEG3 gene expression in human pituitary tumors. J Clin Endocrinol Metab 90:2179-2186, 2005

37. Zhou Y, Zhang X, Klibanski A: MEG3 noncoding RNA: a tumor suppressor. J Mol Endocrinol 48:R45-R53, 2012

38. Zhu H, Guo J, Shen Y, Dong W, Gao H, Miao Y, et al: Functions and mechanisms of tumor necrosis factor- $\alpha$ and noncoding RNAs in bone-invasive pituitary adenomas. Clin Cancer Res 24:5757-5766, 2018

39. Zhu Y, Li T, Chen G, Yan G, Zhang X, Wan Y, et al: Identification of a serum microRNA expression signature for detection of lung cancer, involving miR-23b, miR-221, miR-148b and miR-423-3p. Lung Cancer 114:6-11, 2017

\section{Disclosures}

The authors report no conflict of interest concerning the materials or methods used in this study or the findings specified in this paper.

\section{Author Contributions}

Conception and design: H Wang, Y Zhu. Acquisition of data: D Zhu, Xiao. Analysis and interpretation of data: D Zhu, Xiao, Gao. Drafting the article: D Zhu, Xiao. Critically revising the article: $\mathrm{H}$ Wang, Y Zhu. Reviewed submitted version of manuscript: $\mathrm{H}$ Wang, Z Wang, Y Zhu. Approved the final version of the manuscript on behalf of all authors: H Wang. Statistical analysis: Z Wang, Hu. Administrative/technical/material support: Hu, Duan, Z Zhu, Gao. Study supervision: H Wang, Z Zhu, Y Zhu.

\section{Supplemental Information}

\section{Online-Only Content}

Supplemental material is available with the online version of the article.

Supplemental Tables and Figures. https://thejns.org/doi/ suppl/10.3171/2019.10.JNS191959.

\section{Correspondence}

Haijun Wang: The First Affiliated Hospital of Sun Yat-sen University, Guangzhou, China.wanghaij@mail.sysu.edu.cn. 